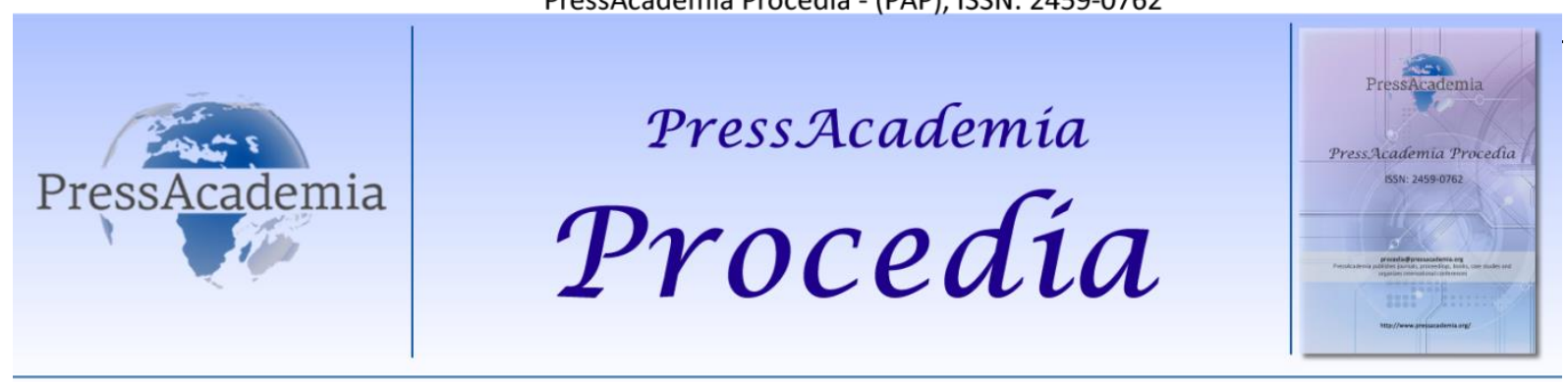

2nd World Conference on Technology, Innovation and Entrepreneurship

May 12-14, 2017, Istanbul, Turkey. Edited by Sefer Şener

\title{
DETERMINATION OF ZIP COEFFICIENTS FOR RESIDENTIAL LOADS
}

\author{
DOI: 10.17261/Pressacademia.2017.587 \\ PAP-WCTIE-V.5-2017(25)-p.176-180
}

\author{
Mehmet Bircan $^{1}$, Ali Durusu ${ }^{2}$, Bedri Kekezoglu ${ }^{3}$, Onur Elma ${ }^{4}$, Ugur Savas Selamogullari ${ }^{5}$ \\ ${ }^{1}$ Yildiz Technical University, Electrical Engineering Department, $\underline{\text { mbircanee@gmail.com }}$ \\ ${ }^{2}$ Yildiz Technical University, Electrical Engineering Department, adurusu@yildiz.edu.tr \\ ${ }^{3}$ Yildiz Technical University, Electrical Engineering Department, bkekez@yildiz.edu.tr \\ ${ }^{4}$ Yildiz Technical University, Electrical Engineering Department, $\underline{\text { onurelma@yildiz.edu.tr }}$ \\ ${ }^{5}$ Yildiz Technical University, Electrical Engineering Department, selam@yildiz.edu.tr
}

\begin{abstract}
In this study, constant-impedance, constant-current and constant-power ZIP models have been analyzed for residental loads. Measurement based approach has been used to obtain ZIP models of selected loads. The measurement-based approach for load modeling is important because it reflects the real dynamics of the system and represents the load characteristics more accurately. The ZIP model is used to estimate the power drawn by the load depending on the voltage changes. Since the ZIP load model is a well-known model that provides power dependence in terms of the physical sense of voltage, studies are taking place in this area. In this study, the voltage and power values of selected residental loads at YTU Smart Home Laboratory are measured. Then, the ZIP coefficients have been calculated by using the least squares algorithm developed in MATLAB. The measured data is compared with the data from the obtained ZIP model for each appliance.
\end{abstract}

Keywords: Load models, ZIP model, residential appliances, least squares method.

\section{INTRODUCTION}

Load modeling is an important issue in power system analysis and it is an essential part of a static and dynamic analysis of power system. Load models are mathematical expressions defining the relationship between active and reactive power and power system voltage and frequency changes. Load models can be considered as the mathematichal representation of relationship between power and voltage. There are mainly two types of load models: static models and dynamic models. Static load models are generally not time-dependent (B. Zhao,2010). Most common static load models are exponential and polynomial load models. Also, a static load model can be defined as a composite ZIP model, which is a well-known model that represents the relationship between applied voltage and power consumption. Dynamic load models can be examined in two parts as input-output load model and physical load model. The most common dynamic load model is the induction motor model (K. Rudion, 2009).

Load characteristics have a significant effect on system performance and it is known that the results of load flow, voltage and transient stability simulations are dependent on the assumed load model (M. Sadeghi, 2009). Thus, the right models for all power system components including loads should be developed for power system analysis. Measurement based and component based approaches are two main approaches used to obtain the load models. Both produce appropriate polynomial or exponential static load models for load flow or stability studies where the load voltage and system frequency do not vary significantly with time (B. Zhao,2010). The polynomial model is commonly named as the ZIP model since the model consists of constant impedance (Z), constant current (I), constant power (P) components. The model parameters are the coefficients defining the proportion of each component (A. Bokhari,2014). Thus, each load has a unique coefficent set that describes the load.

The aim of this work is to obtain the ZIP models of 4 residential appliances at YTU Smart Home Labarotary. For thsi purpose, meassurements are completed to collect data on the power consumption versus applied voltage change. Then, ZIP model 
coefficients are calculated by using the least square algorithm developed in MATLAB. As a result of the study, calculated active power values of examined appliances are compared with actual measurements.

The rest of the paper is organized as follows: Section II presents the mathematical background of ZIP model calculation, Section III describes the evaluated case studies and related results. Section IV finalize the paper with concluding remarks.

\section{DETERMINATION OF ZIP COEFFICIENTS}

Power system loads can represent different characteristics. Accordingly, several different load models could be obtained using mainly three approaches: constant impedance, constant current, or constant power. However, power system loads cannot be always accurately modeled by applying each mentioned approaches individually. Therefore, ZIP models that include all three approaches are used to reach most accurate load models. ZIP models are extensilvely used in power system analyses. In this study, ZIP model coefficients of selected residential appliances in a Smart Home Labaratory at YTU Department of Electrical Engineering are obtained by using experimental results. The steps to determine ZIP coefficients are shown in Fig 1.

Figure 1: The Steps to determine ZIP Coefficients Measurement of $\mathrm{P}_{\mathrm{i}}, \mathrm{V}_{\mathrm{i}}$ values of related appliances Transfer of the measured data to MS EXCEL Reading of $V_{i}, P_{i}$ data by MATLAB Running MATLAB algorithm

Calculation of ZIP coefficients

A programmable AC source is used to obtained voltage-dependent load power measurements. The measurement system is shown in Fig 2. Collected data for each appliance is stored in an excel file and then is processed with MATLAB to calculte ZIP coefficients.

\section{Figure 2: Measurement System}

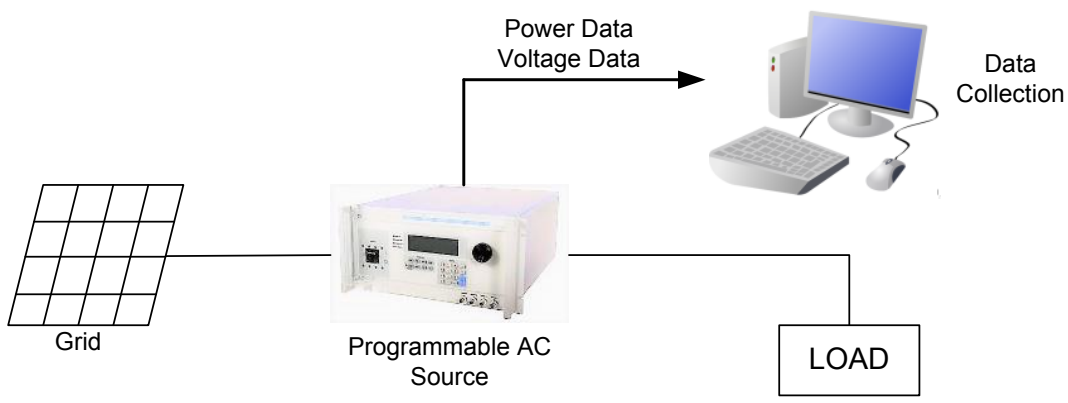

In experiments, the active power consumption of the tested appliance versus $10 \mathrm{~V}$ increments of the applied voltage is collected. The starting value of the applied voltage is $100 \mathrm{~V}$ and the ending value of the applied voltage is $240 \mathrm{~V}$. Using a MATLAB based least squares fit algorithm and measured data, ZIP coefficients of tested appliance are found. Mathematical background of developed algorithm is presented below.

\subsection{ZIP Model}

The ZIP model is a polynomial expression which describes the relationship between the applied voltage and constant impedance, constant current, and constant power components (K. Rudion, 2009). The ZIP model also reveals the quadratic, linear and constant change of the power curve of an element according to voltage (F. L. Quilumba, 2011). Active power and reactive power expressions of a ZIP model are given in Eq. 1 (K. Rudion, 2009):

$$
P=P_{0}\left[Z_{p}\left(\frac{V}{V_{0}}\right)^{2}+I_{p}\left(\frac{V}{V_{0}}\right)+P_{p}\right]
$$




$$
Q=Q_{0}\left[Z_{q}\left(\frac{V}{V_{0}}\right)^{2}+I_{q}\left(\frac{V}{V_{0}}\right)+P_{q}\right]
$$

Here, $P$ and $Q$ are active and reactive power corresponding to the operating voltage $(V), P_{0}$ and $Q_{0}$, are active and reactive power corresponding to nominal voltage $\left(V_{0}\right) ; Z_{p}, I_{p}$ and $P_{p}$ are ZIP coefficients of active power component; $Z_{q}, I_{q}$ and $P_{q}$ are ZIP coefficients of reactive power component.

\subsection{Least Squares Method}

Diffirent algorithms can be used to obtain ZIP coefficients based on measured voltage-power values. In this study, Least Squares Method (LSM) is employed due to its simplicity. The objective function is shown in Eq. 2.

$$
\lambda=\sum_{i=1}^{n}\left(Z_{p}\left(\frac{V_{i}}{V_{0}}\right)^{2}+I_{p}\left(\frac{V_{i}}{V_{0}}\right)+P_{p}-\frac{P_{i}}{P_{0}}\right)
$$

Here, $V_{i}$ and $P_{i}$ are measured values of voltage and active power, respectively. To solve Least Squares problem, partial derivatives of objective function for each variable should be equal to zero. Thus, three equations are obtained for three ZIP coefficients. The solution is achieved by solving these three equations. The solution matrix is given in Eq. 3 ( $M$. Sadeghi,2009).

$$
\left[\begin{array}{ccc}
\sum_{i=1}^{N} V_{i}{ }^{4} & \sum_{i=1}^{N} V_{i}{ }^{3} & \sum_{i=1}^{N} V_{i}^{2} \\
\sum_{i=1}^{N} V_{i}{ }^{2} & \sum_{i=1}^{N} V_{i}{ }^{2} & \sum_{i=1}^{N} V_{i} \\
\sum_{i=1}^{N} V_{i}{ }^{2} & \sum_{i=1}^{N} V_{i} & n
\end{array}\right]\left[\begin{array}{l}
Z_{P} \\
I_{P} \\
P_{P}
\end{array}\right]=\left[\begin{array}{c}
\sum_{i=1}^{N} P_{i} V_{i}^{2} \\
\sum_{i=1}^{N} P_{i} V_{i} \\
\sum_{i=1}^{N} P_{i}
\end{array}\right]
$$

\section{FINDINGS AND DISCUSSIONS}

Three different lighting equipment, incandescent lamp, compact fluorescent lamp and LED lamp, and a kettle are selected as residential appliances and active power versus $10 \mathrm{~V}$ voltage increments between $100 \mathrm{~V}$ and $240 \mathrm{~V}$ are measured and stored within the scope of this study. Then, measured data is processed for each appliance using the LSM algorithm developed in MATLAB. Calculated ZIP coefficients of each appliance are summarized in Table 1.

Table 1: ZIP Coefficients for Each Appliance

\begin{tabular}{lcccc}
\hline Appliance & $\mathbf{Z}$ & $\mathbf{~}$ & $\mathbf{P}$ & $\mathbf{Z + 1 + P}$ \\
\hline Incandescent lamp & 0.49 & 0.59 & -0.08 & 1 \\
LED lamp & 0.73 & -1.7 & 1.97 & 1 \\
Compact fluorescent lamp & 0.11 & 0.64 & 0.25 & 1 \\
Kettle & 0.95 & 0.07 & -0.02 & 1 \\
\hline
\end{tabular}

As seen, constant impedance model is convenient for the kettle while it is more appropriate to use ZIP model for other appliances. Comparision of the actual measurements and obtained ZIP model ouputs are given in Fig. 3. 
Figure 3: Comparison of ZIP Model Outputs with Measured Values
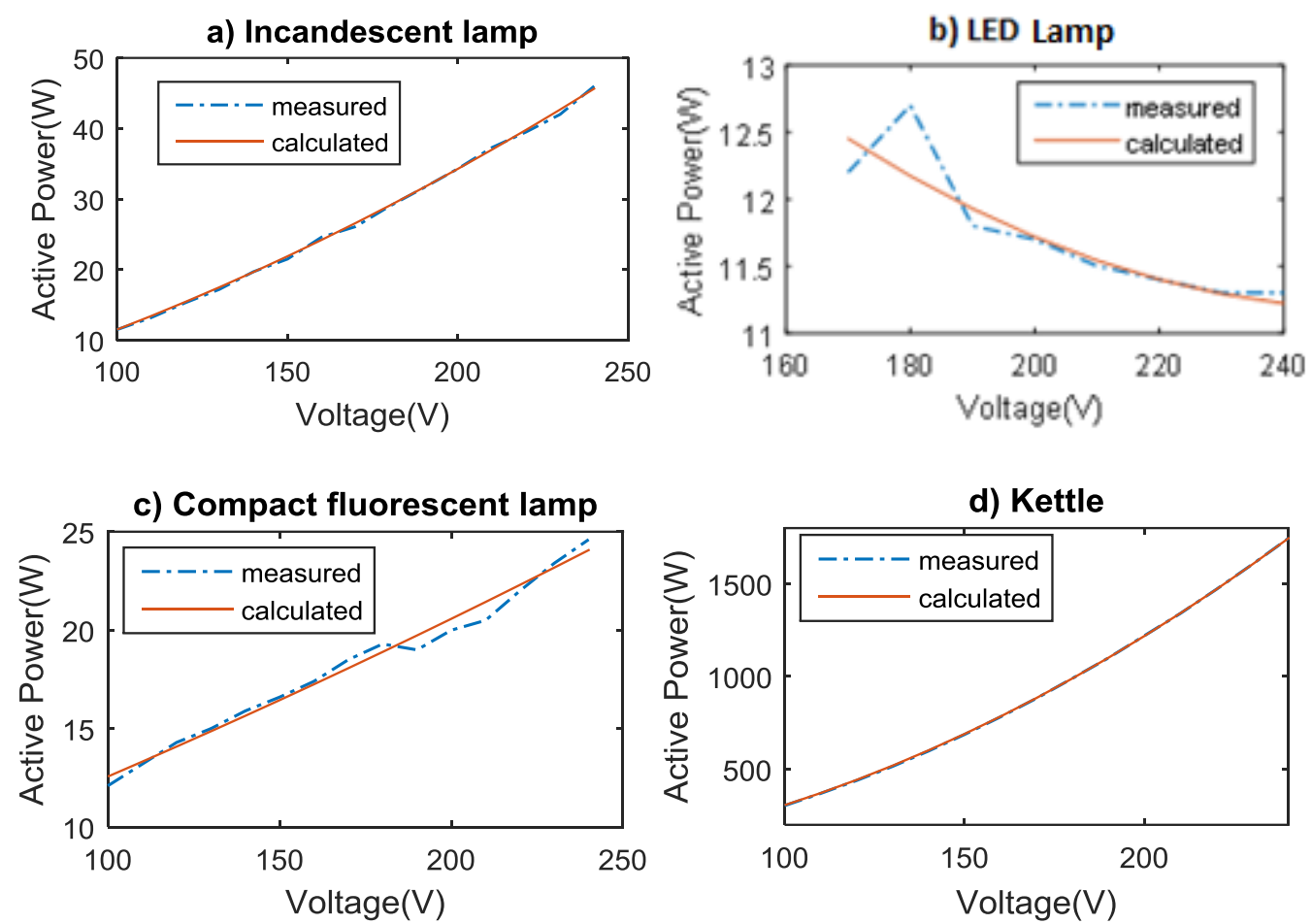

Calculated and measured values of incandescent lamp are shown in Fig. 3a. Nominal voltage of lamp is $220 \mathrm{~V}$ and the active power at nominal voltage is $39.8 \mathrm{~W}$. By examining Figure $3 \mathrm{a}$, it is seen that calculated values are very close to the measured values and active power characteristic of the incandescent lamp can be correctly modeled with the ZIP model.

Power changes of LED lamp is given in Fig. $3 \mathrm{~b}$. Nominal voltage of LED lamp is $220 \mathrm{~V}$ and the active power at nominal voltage is $11.4 \mathrm{~W}$. Fig. $3 \mathrm{~b}$ shows that calculated values are very close to the measured values at above $200 \mathrm{~V}$ voltage level. For the practical purposes, the obtained ZIP model for the LED lamp can be a valid model considering that the nominal voltage of appliances in Turkey is around 200-220V level. However, it is seen that the difference between the measured and calculated power values is rising at low voltage levels.

Calculated and measured values of compact fluorescent lamp are compared in Fig. 3c. Nominal voltage of the lamp is $220 \mathrm{~V}$ and the active power at nominal voltage is $22.3 \mathrm{~W}$. It is seen that the measured and calculated values are very close.

Power changes of the kettle is given in Fig. 3d. Nominal voltage of the kettle is $220 \mathrm{~V}$ and the active power at nominal voltage is $1470 \mathrm{~W}$. It has been observed that the measured and calculated curves for the kettle overlapping.

\section{CONCLUSIONS}

Load models have significant importance for accurate power system analysis. Power system loads can be modeled in different ways. In this study, ZIP coefficients of residential loads at YTU Smart Home Labarotory are obtained by using actual measurement data. Primarily, power measurements are performed at the operating voltage ranges of considered loads, Obtained data is then processed with help of a solution algorithm developed in MATLAB and ZIP coefficients of the selected appliances are calculated. Examination of ZIP coefficient results shows that the kettle is suitable for constant impedance model, however it is observed that ZIP model is more appropriate for the other appliances. When the ZIP model output and measured data are compared for each appliance, it is seen that calculated and measured values of incandescent lamp and compact fluorescent lamp are very close. The power characteristic for the LED lamp is accurate at voltages above 200V, however deviations are observed at low voltages. It is seen that the measured and calculated power curves overlap for the kettle. 


\section{REFERENCES}

B. Zhao, Y. Tang, W. Zhang, and Q. Wang, "Modeling of common load components in power system based on dynamic simulation experiments," in Proc. Int. Conf. Power Syst. Technol., 2010, pp. 1-7.

K. Rudion, H. Abildgard, Z.A. Styczynski "Non-linear load modeling - Requirements and preparation for measurement," Power \& Energy Society General Meeting, 2009, pp. 1-5.

M. Sadeghi and G. A. Sarvi, "Determination of ZIP parameters with least squares optimization method," in Elect. Power Energy Conf., 2009, pp. 1-6.

A. Bokhari, A. Alkan, R. Dogan, M. D.-Aguiló, F. León, D. Czarkowski, L. Birenbaum, A. Noel, R. E. Uosef, " Experimental Determination of the ZIP Coefficients for Modern Residential, Commercial, and Industrial Loads", IEEE Transactıons On Power Delıvery, Vol. 29, No. 3, June 2014.

F. L. Quilumba, W. Lee, H. Huang, D. Y. Wang, and R. L. Szabados, "Load model development for next generation appliances," in Proc. Ind. Appl. Soc. Annu. Meeting, 2011, pp. 1-7. 